\title{
Comparison of GeneXpert and line probe assay for detection of Mycobacterium tuberculosis and rifampicin-mono resistance at the National Tuberculosis Reference Laboratory, Kenya
}

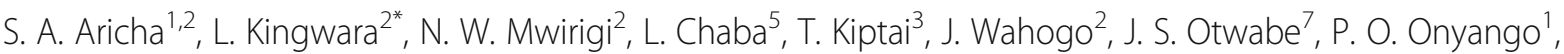 \\ M. Karanja ${ }^{6}$, C. Ayieko ${ }^{1}$ and S. W. Matu ${ }^{4}$
}

\begin{abstract}
Background: The dual challenge of low diagnostic sensitivity of microscopy test and technical challenge of performing a TB culture test poses a problem for case detection and initiation of Tuberculosis (TB) second-line treatment. There is thus need for a rapid, reliable and easily accessible assay. This comparative analysis was performed to assess diagnostic performance characteristics of GeneXpert MTB/RIF and Line Probe Assay (LPA).

Methods: Three hundred twenty nine sputum samples of patients across the 47 counties in Kenya suspected to have drug resistant TB were picked and subjected to GeneXpert, LPA and Culture MGIT at the National TB Reference Laboratory. Sensitivity, specificity and predictive values were then determined to assess the performance characteristics of the various assays

Results: Against culture MGIT as the gold standard for TB diagnosis, GeneXpert had a sensitivity, specificity, positive predictive value, and negative predictive value of 78.5, 64.9, 59.4 and 82.2\% respectively while LPA had 98.4, 66.0, 65.4 and $98.4 \%$. For diagnosis of rifampicin mono-resistance GeneXpert had a moderate agreement (Kappa 0.59, $P<0.01$ ) (sensitivity $62.50 \%$, specificity $96.50 \%$ ) while LPA that had almost perfect agreement (Kappa $=0.89, p<0.01$ ) with a (sensitivity $90.0 \%$ and specificity $99.1 \%$ ).
\end{abstract}

Conclusion: LPA has a better performance characteristic to GeneXpert and an alternative to culture with regards to detection of RIF's mono-resistance.

Keywords: LPA, GeneXpert, Sensitivity, Specificity, Drug-resistant TB

\section{Background}

Tuberculosis (TB) causes substantial morbidity and mortality [1]. About 10.0 million TB cases and 1.4 million deaths worldwide were reported in 2018 [2]. In the same year, 480,000 cases of multidrug-resistant TB (MDR-TB) and 100,000 cases of rifampicin monoresistant TB were reported. Kenya is among the 22 countries with the highest TB burden globally, with a national TB case prevalence of 558 per 100,000 people as per the Kenya

* Correspondence: leonard.kingwara@gmail.com

${ }^{2}$ National Public Health Laboratories, Nairobi, Kenya

Full list of author information is available at the end of the article
National TB prevalence survey 2016 [3, 4]. A key component in TB control and management is rapid diagnosis [5]. Tuberculosis diagnosis is routinely done using microscopy in middle and low-income countries since it is readily available. Microscopy identifies Mycobacterium tuberculosis bacilli which is treatable with first-line AntiTB drugs. Its sensitivity in TB detection is at $60 \%$ as per the Kenya national TB case prevalence survey of 2016 resulting into a high number of missed cases. This results into a continuous transmission especially among the missed cases. Moreover TB microscopy has a low

(c) The Author(s). 2019 Open Access This article is distributed under the terms of the Creative Commons Attribution 4.0 International License (http://creativecommons.org/licenses/by/4.0/) which permits unrestricted use, distribution, and reproduction in any medium, provided you give appropriate credit to the original author(s) and the source, provide a link to the Creative Commons license, and indicate if changes were made. The Creative Commons Public Domain Dedication waiver (http://creativecommons.org/publicdomain/zero/1.0/) applies to the data made available in this article, unless otherwise stated. 
sensitivity in the detection of TB in children and patients with extra- pulmonary TB [1].

Drug resistance TB is transmissible and results mainly from sub-optimal TB treatment. The diagnostic tools available for its detection are limited and often less sensitive and poorly adaptable for poor resource settings [1]. This results to large proportion of drug resistance TB cases remaining undiagnosed leading to continuous transmission and increased cost of treatment and management. Currently, TB culture remains the gold standard for drug resistance TB diagnosis although it is more expensive and takes not less than 4 weeks to get results [6].

The molecular-based methods detect Mycobacterium tuberculosis nucleic acid materials in sputum samples and the mutations resulting into anti TB drugs resistance. GeneXpert and LPA methods are both capable of detecting mutations causing resistance against Rifampicin. In addition, LPA can also detects mutations related to isoniazid drug [5]. Though widely distributed in the Kenyan health care system, the performance of these two assays have not been evaluated to determine their performance in the detection of TB and associated drug resistance.

Early diagnosis of TB resistance is important for optimal management of drug-resistant cases; this necessitates rapid drug susceptibility testing in the management of MDR/XDR TB [6, 7]. The standard DST test is a twostep procedure involving culture and sensitivity testing. This takes a long time, first to isolate a culture and then to perform drug susceptibility testing (indirect DST). If DST could be set up at the same time as when a processed specimen is inoculated in solid and or liquid medium (direct DST), it could save significant time for the detection of drug resistance.

In Kenya, direct DST using the conventional solid medium has been established $[8,9]$. The only disadvantage is that it takes a long time to obtain results on solid medium, as the growth rate on such media is lower. This impacts negatively to critical patients who are in need of a shorter result turnaround time for patient management.

The study thus determined the sensitivity, specificity, positive and negative predictive values of GeneXpert and LPA in TB diagnosis using liquid culture as the gold standard. In addition, we assessed the sensitivity, specificity, positive and negative predictive values of GeneXpert and LPA in Rifampicin mono resistance detection using liquid culture as the gold standard.

\section{Methods}

We analysed 306 samples between November 2016 and March 2017. A systematic random sampling procedure was used to select sputum samples from a pool received from various diagnostic sites located in the 47 counties. An average of ten samples was picked from all samples received at NTRL during each day for a period of 33 days to obtain 329 samples. To ensure equal chance of every sample received being selected, all the samples received in a day were divided by 10 to get the interval of picking.

\section{Sample collection and transportation}

Suspected MDR-TB patients from clinical diagnosis and patient result history review were given a sputum collection container with instructions on how to collect and deliver a sputum sample to the facility. The sputum sample together with the patient request form from each diagnostic site was packed in a standard triple packaging container and an ice bag inserted to keep the sample at 2$8{ }^{\circ} \mathrm{C}$. All the samples were transported by a registered courier to NTRL for culture, drug susceptibility and molecular diagnostic testing.

\section{Sample reception and processing}

At NTRL, sputum samples together with the lab request form were received and checked for completeness in filling the laboratory request form, correct sample tube labelling and leakage. Those accepted were given laboratory number for processing. All acceptable sputum samples were decontaminated using the $N$-acetyl-l-cysteine-sodium citrate- $\mathrm{NaOH}$ (NALC$\mathrm{NaOH})$ method. Samples were decanted following centrifugation at $3000 \mathrm{~g}$ for $15 \mathrm{~min}$, and the pellets were re-suspended to make $3 \mathrm{ml}$ using phosphate buffer solution. Four $0.5 \mathrm{ml}$ aliquots from each sample were used in florescent microscopy, MGIT960 culture, GeneXpert, and LPA. Another $1 \mathrm{ml}$ aliquot of each sample was stored at $-80^{\circ} \mathrm{C}$ as back up.

\section{MGIT960 culture and drug susceptibility testing (SIRE MGIT-DST)}

A $500-\mu \mathrm{l}$ aliquoted sample were incubated in $\mathrm{Myco}$ bacterium growth indicator tubes (MGIT) in the BACTEC machine (BACTEC $^{\mathrm{mm}}$ MGIT $^{\mathrm{mm}} 960$ System, Series 1300 A2, Becton Dickson and Company, MA, USA) for a maximum of 42 days (6 weeks) from initial incubation date. The MGIT tubes in the BACTEC machine were flagged by green light for no growth and red light for growth of Mycobacterium, on the front drawer of the BACTEC MGIT 960 machine. Cultures that showed growth were confirmed for Mycobacterium tuberculosis by use of brain heart infusion agar (BHI), Ziehl Neelsen stain and capillia technique and subjected to drug susceptibility testing (DST) against Isoniazid, Rifampicin, Streptomycin and Ethambutol (SIRE). To control tubes, $0.5 \mathrm{ml}$ of Growth Control working solution was added into 
labelled drug free Mycobacterium growth indicator tubes, and to all the other drugs containing tubes labelled S (streptomycin), I (isoniazid), R (rifampicin) and $\mathrm{E}$ (ethambutol). When the growth control tubes reached a growth unit of 400 or more the instrument indicated that the test was complete and interpreted the results as resistant or susceptible. The tubes were removed from the machine after being scanned and printed. The final pattern of susceptibility testing was manually interpreted per sample as fully susceptible, mono-resistant, poly-resistant or multidrug resistant. To assure the test quality a set of H37RV controls were included in all the run.

\section{Line probe assay}

The line probe assay (LPA), based on strip technology was used to diagnose TB and detect RIF as well as Isoniazid (INH) resistance due to mutations in rpo $\beta$, and both inhA and katG genes. The test was performed according to the manufacturer's protocol (Hain Life Science $\mathrm{GmbH}$, Nehren, Germany). The method involved three processes: DNA extraction, multiplex PCR amplification, and reverse hybridization. The DNA strip was removed from the tube and marked as per the number of samples. It was then added to each well containing $20 \mu \mathrm{l}$ of corresponding amplified DNA sample with coloured part facing up. The well was placed in the twincubator and hybridization procedure was initiated. Hybridization occurred by pre-warming the hybridization buffer to $45^{\circ} \mathrm{C}$ in water bath for $15 \mathrm{~min}$ in the twincubator machine (Hain Life Science $\mathrm{GmbH}$, Nehren, Germany). Denaturing solution, $20 \mu \mathrm{l}$ was pipetted to each of the tray that was used, and then $1 \mathrm{ml}$ of rinse solution added per well and incubated for $1 \mathrm{~min}$. The well was removed and rinsed with a rinse solution. One millilitre of the conjugate was added into each well, then incubated for $30 \mathrm{~min}$, removed and washed with rinse solution. Finally, $1 \mathrm{ml}$ of the substrate was added into the well and incubated for $10 \mathrm{~min}$ and then washed twice with distilled water. The strips were then left to dry and results scanned and interpreted by the Hain Life Science $\mathrm{GmbH}$, Nehren, Germany machine as either Mycobacterium tuberculosis detected, resistant, sensitive or invalid.

\section{GeneXpert MTB/RIF}

The GeneXpert MTB/RIF (Cepheid, Sunnyvale, CA) test was performed as per the manufacturer's instruction. Aliquots of decontaminated samples were taken out of $4{ }^{\circ} \mathrm{C}$ storage, together with the sample reagent buffer containing $\mathrm{NaOH}$ and isopropanol were mixed at the ratio of 1:3 followed by incubation at room
Table 1 Distribution of participants' Age, Gender, and Smear concentration microscopy results

\begin{tabular}{lll}
\hline & FREQUENCY/MEAN & PERCENT/SD \\
\hline AGE & Mean is 36.8 & 13.3 \\
GENDER & & \\
FEMALE & 108 & 35.3 \\
MALE & 198 & 64.7 \\
SMEAR CONCENTRATION & \\
$1+$ & 48 & 15.7 \\
$2+$ & 56 & 18.3 \\
$3+$ & 41 & 13.4 \\
$4 B$ & 1 & 0.3 \\
5B & 3 & 1.0 \\
10B & 1 & 0.3 \\
NEG & 120 & 39.2 \\
$6 B$ & 36 & 11.8 \\
\hline
\end{tabular}

temperature for $15 \mathrm{~min}$. Two milliliters of the sample were then transferred into the GeneXpert MTB/RIF cartridge (Cepheid, Sunnyvale, CA) and loaded into the GeneXpert MTB/RIF (Cepheid, Sunnyvale, CA). The results were generated after 2 hours, reported as both $M$. tuberculosis negative or positive, and whether those positive were RIF susceptible or resistant.

\section{Ethical consideration}

Ethical approval for this study was obtained from Amref Health Africa Ethics and Scientific Review committee. The samples were assigned unique study codes and delinked with the patient maintaining age and sex as the only socio-demographic data. The study did not alter the original patient results in any way.

\section{Statistical analysis}

Categorical and numerical variables were summarized using frequency (percentage) and mean (SD) respectively.

Table 2 M. tuberculosis detection by GeneXpert and LPA against Culture MGIT using smear positive samples

\begin{tabular}{|c|c|c|c|}
\hline & \multicolumn{2}{|c|}{ Smear positive } & \multirow[b]{2}{*}{ Total $(=186)$} \\
\hline & $\begin{array}{l}\text { Detected } \\
(n=119)\end{array}$ & $\begin{array}{l}\text { Not Detected } \\
(n=67)\end{array}$ & \\
\hline \multicolumn{4}{|l|}{ Gene Xpert } \\
\hline Detected & 94 & 48 & $142(76.3 \%)$ \\
\hline Not Detected & 25 & 19 & $44(23.7 \%)$ \\
\hline \multicolumn{4}{|l|}{ LPA } \\
\hline Detected & 118 & 49 & 167 (89.8\%) \\
\hline Not Detected & 1 & 18 & 19 (10.2\%) \\
\hline
\end{tabular}


Table 3 Performance of GeneXpert and LPA tests compared to Culture MGIT in detecting M. tuberculosis using smear positive samples

\begin{tabular}{lllcll}
\hline & Sensitivity (\%) & Specificity (\%) & \multicolumn{2}{l}{ Predictive values (\%) } & Kappa \\
\cline { 4 - 5 } & & & Positive & Negative & $0.079(P=0.257)$ \\
\hline Gene Expert & $66.2(57.8-73.9)$ & $43.2(28.4-59.0)$ & $79(73.9-83.3)$ & $28.4(20.8-37.4)$ & $0.309(p<0.001)$ \\
LPA & $99.2(95.4-100.0)$ & $26.9(16.8-39.1)$ & $70.7(67.5-73.6)$ & $94.7(71.1-99.3)$ & \\
\hline
\end{tabular}

Sensitivity, specificity and predictive values were calculated to compare the performances between tests. Agreements of different tests were assessed by Cohen's Kappa statistics. A test with $P \leq 0.05$ was considered statistically significant. The precision of the estimates was reported using 95\% confidence intervals (95\% CI). Statistical analyses were done using $\mathrm{R}$.

\section{Results}

Three hundred and twenty-nine sputum samples of patients suspected to have drug resistant $\mathrm{TB}$ were picked and subjected to GeneXpert, LPA and Culture MGIT. Out of 329 samples processed, 23 samples were excluded; 10 had Mycobacterium other than $M$. tuberculosis; 4 were contaminated; 4 were invalid; 3 had errors; 2 were indeterminate. The remaining three hundred and six (306) samples were analysed. Of the 306 samples, 108 (35.29\%) were from female and 198 (64.71\%) from male participants. The mean age of study participants who provided sputum samples used in this study was $36.86(\mathrm{SD}=13.3)$ years. Of the 306 samples tested for smear concentration microscopy, 39.2\% were smear negative (). For the smear positive samples, the following bacillary loads were observed: $1+$ in 48 (15.7\%), $2+$ in 56 (18.3\%) and 3+, 41 (13.4\%), 4B, 5B and $10 \mathrm{~B}$ were observed in just 5 samples while $6 \mathrm{~B}$ results were observed in 36 (11.8\%) samples (Table 1).

\section{Diagnosis of tuberculosis}

To compare the performance of GeneXpert and LPA on smear positive results, 186 samples were used. GeneXpert detected 142 (76.5\%) and LPA detected $167(89.8 \%)$ samples as positive. In terms of sensitivity, specificity, and predictive values, GeneXpert recorded sensitivity of $62.2 \%$ and specificity of $43.2 \%$ with positive and negative predictive value of 79.0 and $28.4 \%$ respectively. LPA reported sensitivity and specificity of 99.2 and $26.9 \%$ respectively with positive and negative predictive value of 70.7 and $94.7 \%$ respectively (Tables 2 and 3). Of 306 samples tested, Culture MGIT detected M.tuberculosis in 121 (39.5\%) samples, GeneXpert detected M.tuberculosis in 160 (52.3\%), and LPA detected M.tuberculosis in 182 (59.5\%). (Table 4). Using Culture MGIT as the gold standard, the GeneXpert correctly identified $M$. tuberculosis in 95 of 121 Culture MGIT positive samples while LPA correctly identified M. tuberculosis in 119 of 121 Culture MGIT positive samples. These translate to sensitivity, specificity, positive predictive value, and negative predictive value of $78.5,64.9,59.4$ and $82.2 \%$ respectively for the GeneXpert and 98.4, 66.0, 65.4 and $98.4 \%$ respectively for the LPA (Table 5).

The 121 Culture MGIT positive samples were subjected to the drug susceptibility testing (DST) against Isoniazid, Rifampicin, Streptomycin and Ethambutol. 9 (7.4\%), 12 (9.9\%), 10 (8.3\%), and 7 (5.8\%) samples were found to be resistant to Streptomycin, Isoniazid, Rifampicin and Ethambutol respectively. $4.1 \%$ of the samples were resistant to all the four antibiotics and 86.6 of the samples were sensitive to all the antibiotics. (Table 6).

For assessing the performance of GeneXpert and LPA tests in detecting Rifampicin and Isoniazid resistance, pairwise undetected results from the Culture MGIT, GeneXpert and LPA tests were excluded. Culture MGIT and GeneXpert were then compared in 95 samples while LPA and Culture MGIT were compared in 119 samples. Culture MGIT drug susceptibility testing was used as the gold standard. GeneXpert and LPA had a sensitivity value of $91.6 \%$ (Table 7). There was a moderate agreement $($ Kappa $=0.59, P<$ 0.01 ) between culture and Gene expert in detecting Rifampicin mono-resistance (sensitivity $=62.50 \%$, specificity $=96.50 \%$ ) and an almost perfect agreement (Kappa $=0.89, p<0.01)$ between Culture and LPA

Table 4 M. tuberculosis detection by GeneXpert and LPA against Culture MGIT

\begin{tabular}{llll}
\hline & \multicolumn{2}{l}{ Culture MGIT } & \\
\cline { 2 - 3 } & $\begin{array}{l}\text { Detected } \\
(n=121)\end{array}$ & $\begin{array}{l}\text { Not Detected } \\
(n=185)\end{array}$ & Total (=306) \\
\hline $\begin{array}{l}\text { Gene Xpert } \\
\text { Detected }\end{array}$ & 95 & 65 & $160(52.3 \%)$ \\
Not Detected & 26 & 120 & $146(47.7 \%)$ \\
LPA & 119 & 63 & $182(59.5 \%)$ \\
Detected & 2 & 122 & $124(40.5 \%)$ \\
Not Detected & & & \\
\hline
\end{tabular}


Table 5 Performance of GeneXpert and LPA tests compared to Culture MGIT in detecting M. tuberculosis

\begin{tabular}{llllll}
\hline & Sensitivity (\%) & Specificity (\%) & \multicolumn{2}{l}{ Predictive values (\%) } & \multicolumn{2}{l}{ Kappa } \\
\cline { 4 - 5 } & & & Positive & Negative & $0.411(P<0.01)$ \\
\hline GeneXpert & $78.5(70.1-85.5)$ & $64.86(57.5-71.7$ & $59.4(54.1-64.5)$ & $82.2(76.4-86.8)$ & $0.591(p<0.01)$ \\
LPA & $98.4(94.2-99.8)$ & $66.0(58.6-72.7)$ & $65.4(60.7-69.8)$ & $98.4(93.9-99.6)$ & 0 \\
\hline
\end{tabular}

$($ sensitivity $=90.0 \%$ and specificity $=99.1 \%) \quad($ Table 8$)$. The agreement between Culture MGIT and LPA in detecting Isoniazid (KatG and INHA) resistance was substantial (Kappa $=0.758 p<0.001$ ) with sensitivity and specificity values being 91.7 and $95.3 \%$ respectively (Tables 9 and 10).

\section{Discussion}

Resurgence and rapid spread of $\mathrm{TB}$ and drug resistant $M$. tuberculosis over the recent years raises an urgent need to find an efficient rapid assay for diagnosis and detection of drug resistant TB [10]. No study has been carried out in Kenya to compare the performance of both GeneXpert and LPA. In this study, the performance of GeneXpert MTB/RIF and LPA in detecting TB and Rifampicin mono-resistance was compared to that of liquid culture as the gold standard.

Using smear as the gold standard for diagnosing $\mathrm{TB}$, GeneXpert MTB/RIF, had a diagnostic sensitivity, specificity, positive predictive value and negative predictive values comparable to the Thibela TB' study that yielded a sensitivity, specificity, PPV and NPV of 59.8\%, 49/7, 79.2 and $31.5 \%$ [11-13]. Similar studies have yielded

Table 6 Antibiotic resistant detection by DST MGIT on positive

\begin{tabular}{|c|c|c|}
\hline \multicolumn{3}{|l|}{$N=121$} \\
\hline & Frequency & Percent \\
\hline \multicolumn{3}{|l|}{ DST_1ST_STREPTOMYCIN } \\
\hline RESISTANT & 9 & 7.4 \\
\hline SENSITIVE & 112 & 92.6 \\
\hline \multicolumn{3}{|l|}{ DST_1ST_ISONIAZID } \\
\hline RESISTANT & 12 & 9.9 \\
\hline SENSITIVE & 109 & 90.1 \\
\hline \multicolumn{3}{|l|}{ DST_1ST_RIFAMPICIN } \\
\hline RESISTANT & 10 & 8.3 \\
\hline SENSITIVE & 111 & 91.7 \\
\hline \multicolumn{3}{|l|}{ DST_1ST_ETHAMBUTOL } \\
\hline RESISTANT & 7 & 5.8 \\
\hline SENSITIVE & 114 & 94.2 \\
\hline RESISTANT TO ALL GENES & 5 & 4.1 \\
\hline SENSITIVE TO ALL GENES & 105 & 86.8 \\
\hline
\end{tabular}

similar results. LPA had a better diagnostic performance characteristic compared to GeneXpert when using smear microscopy as the gold standard with results comparable to the South African meta-analysis study which yielded a sensitivity, specificity, positive predictive value and negative predictive values of 99.1, 28.4, 69.9 and $94.6 \%$ respectively [14-18]. Studies in similar settings have also reported same results [19-21].

GeneXpert performance when MGIT was used as the gold standard, gave result similar to other studies that had Sensitivity, specificity, positive predictive value, and negative predictive values of MTB at 62.6\%(55.2, 69.5), 99.6\% (99.4, 99.7), 81.3\% (73.9, $87.3)$, and 98.9 (98.6, 98.8), while Kappa agreement was $98.5 \%(98.2,98.8)$. Similar results have also been seen among HIV-infected adults enrolling in an ART clinic whose sputum was tested by GeneXpert regardless of symptoms [21]. Our LPA findings compares to the sensitivity and specificity of LPA diagnostic platform in South African and South American was 81 and $100 \%$ respectively [22]. In India two studies showed a sensitivity and specificity of 96 and 99\% respectively in 248 smear positive patients [13] and, sensitivity and specificity of 68.4 and $89.3 \%$ respectively from 213 sputum smear negative samples [23]. Similarly, a study in high risk MDR-TB population in Taiwan also reported a sensitivity and specificity of 85.9 and $65.7 \%$ respectively [24]. Also, in Ethiopia, a study on 274 presumptive MDR-TB patients both smear positive and negative, reported sensitivity and specificity of 96.4 and $100 \%$ from smear positive samples, 77.8 and $97.2 \%$ smear negative samples respectively [11]. It appears that failure to separate the

Table 7 Detection of rifampicin resistance by GeneXpert and LPA against Culture MGIT DST

\begin{tabular}{llll}
\hline & \multicolumn{2}{l}{ DST_1ST_RIF } & \\
\cline { 2 - 4 } & Resistant $(n=8)$ & Sensitive $(n=89)$ & Total $(n=95)$ \\
\hline GENEXPERT RIF & & 3 & $8(8.4 \%)$ \\
RESISTANT & 5 & 34 & $87(91.6 \%)$ \\
SENSITIVE & 3 & 84 & Total $(n=119)$ \\
LPA RIF & Resistant $(n=10)$ & Sensitive $(n=109)$ & $10(8.4 \%)$ \\
RESISTANT & 9 & 1 & $109(91.6 \%)$ \\
SENSITIVE & 1 & 108 & \\
\hline
\end{tabular}


Table 8 Performance of Gene expert and LPA test as compared to DST in detecting RIF resistance

\begin{tabular}{llllll}
\hline & Sensitivity & Specificity & \multicolumn{2}{l}{ Predictive values } & Kappa \\
\cline { 3 - 5 } & & & Positive & Negative & $0.59(P<0.01)$ \\
\hline GeneXpert & $62.5(24.5-91.5)$ & $96.6(90.3-99.3)$ & $62.5(32.7-85.1)$ & $96.6(92.098 .6)$ & $0.89(P<0.01)$ \\
LPA & $90.0(55.5-99.8)$ & $99.1(95.0-100)$ & $90.0(55.9-98.5)$ & $99.1(94.4-99.9)$ & \\
\hline
\end{tabular}

samples as per the smear results did not affect the sensitivity but affected the specificity in this study.

There was a moderate agreement (Kappa $=0.59, P<$ 0.01 ) between culture and Gene expert in detecting Rifampicin resistance (sensitivity $=62.50 \%$, specificity $=$ 96.50\%) when MGIT was used as the reference assay. This was comparatively lower than LPA that had almost perfect agreement (Kappa $=0.89, \quad p<0.01)$ between MGIT Culture and LPA (sensitivity $=90.0 \%$ and specificity $=99.1 \%$ ) for detecting Rifampicin resistance and a substantial agreement (Kappa $=0.758 p<0.001$ ) with sensitivity and specificity values being 91.7 and $95.3 \%$ respectively for detecting in detecting Isoniazid (KatG and INHA) resistance.

GeneXpert MTB/RIF sensitivity, specificity, positive and negative predictive values for Rifampicin mono resistance detection of 62.50 and $96.50 \%, 62.50$ and $96.60 \%$ respectively compares with a similar study in South Africa which recorded sensitivity and specificity of 79 and $94 \%$ respectively [25]. It was notable that our study reported lower sensitivity compared to the South African study and a similar one within the Asian population which had a pooled sensitivity of $68-100 \%$ [26]. Previous studies have suggested reasons for these, for example discrepancy in the volume of the sample discharged into the cartridge [27] and failure to separate samples based on smear results because smear positive samples have been reported with higher sensitivity when compared to samples which are smear negative [11].

The sensitivity of Rifampicin mono resistance using LPA observed in this study is corresponding to other

Table 9 Detection of Isoniazid (INH) resistance by LPA against Culture MGIT DST

\begin{tabular}{clll}
\hline \multicolumn{5}{c}{ DST_1ST_ISONIAZID } \\
\cline { 2 - 4 } & Resistant $(n=12)$ & Sensitive $(n=107)$ & Total $(n=119)$ \\
\hline KATG & & $11(9.2 \%)$ \\
RESISTANT & 8 & 3 & $108(90.8 \%)$ \\
SENSITIVE & 4 & 104 & \\
NHA & & & $5(4.2 \%)$ \\
RESISTANT & 3 & 2 & $114(95.8 \%)$ \\
SENSITIVE & 9 & 105 & $16(13.4 \%)$ \\
KATG AND INHA & & & $103(86.6 \%)$ \\
RESISTANT & 11 & 5 & \\
SENSITIVE & 1 & 102 & \\
\hline
\end{tabular}

similar studies. Two case examples from South African and South American population using the smear positive samples, had a sensitivity and specificity of 92 and $97 \%$ [22]. This is corresponding to similar findings from a study based on high risk MDR- TB set up of Taiwan which reported a sensitivity of $96 \%$ [24]. In addition, a study in New Delhi recorded a sensitivity and specificity of 97.6 and $94.4 \%$ when they used LPA in smear positive samples [28] similarly to the South Africa study reported a sensitivity and specificity of 97.7 and $91.8 \%$ respectively [29]. In other studies done in East African countries, the sensitivity and specificity of Rifampicin mono resistance detection was 96.4 and $100 \%$ among smear positive samples and 77.8 and $98.2 \%$ among smear negative samples in Ethiopia [11] and 100 and $96.1 \%$ among smear positive population in Uganda [30]. Predictive values reported in RIF mono resistance determination by both GeneXpert and LPA was high thus a better diagnostic tool for RIF resistance diagnosis.

This study reported a moderate agreement between GeneXpert MTB/RIF and culture (Kappa Value, 0.4109), and also LPA and culture (Kappa Value, 0.5914). Similarly, there was a moderate agreement between GeneXpert MTB/RIF and culture (Kappa Value, 0.5905) for Rifampicin mono resistance detection while, on the other hand there was a very good agreement between LPA and culture (Kappa Value, 0.8635) for detection of RIF mono-resistance. The current study reported a high agreement similar to what was reported in India, of 100\% between MGIT960 and LPA results but only 64.4\% agreement with GeneXpert MTB/RIF results [31], equally LPA reporting the same with the conventional DST at $96 \%$ in New Delhi [32]. Therefore, the study recommends use of either GeneXpert or LPA for TB detection and LPA for RIF mono-resistant.

Our results slightly vary from similar studies. A case example are the results that showed the sensitivity and specificity of the GeneXpert assay in M. tuberculosis samples from South Africa and Turkey to be $92.7-100 \%$ and $96.3-100 \%$, respectively [21,33-37]. In other areas such as Vietnam and Malaysia, similar values have been reported as 59 and 53\%, respectively which is much lower than our findings $[12,14,21]$. The variations of the assay performance characteristics can be attributed to the geographical features of the sampling locations, differences in sampling method, MDR-TB and mutations on the $r p o B$ gene in populations. 
Table 10 Performance of LPA test as compared to DST in detecting Isoniazid (INH) resistance

\begin{tabular}{llllll}
\hline & Sensitivity & Specificity & \multicolumn{2}{l}{ Predictive values } & Kappa \\
\cline { 4 - 5 } & & & Positive & Negative & $0.663(P<0.01)$ \\
\hline KatG & $66.7(34.9-90.1)$ & $97.2(92.0-99.4)$ & $72.7(44.9-89.7)$ & $96.3(92.1-98.3 \%)$ & $0.319(P<0.01)$ \\
inhA & $25.0(5.5-57.19)$ & $98.1(93.4-99.8)$ & $60(21.7-89.01)$ & $92.11(89.4-94.2)$ & $0.758(p<0.01)$ \\
\hline
\end{tabular}

\section{Conclusion}

In our context, when MGIT is used as the reference assay standard, Gene-Xpert has a better diagnostic performance characteristic than AFB Microscopy but lower when compared to the LPA. In addition, regarding RIF mono-resistance, LPA outperformed GeneXpert MTB/ RIF and thus it is a better alternative to culture with regards to detection of RIF.

\section{Supplementary information}

Supplementary information accompanies this paper at https://doi.org/10. 1186/s12879-019-4470-9.

Additional file 1. Number of samples collected from the 34 counties.

\section{Abbreviations}

AFB: Acid fast bacili; BHI: Brain heart infusion agar; INH: Isoniazid; LPA: Line probe assay; MDR-TB: Multi-drug resistance tuberculosis;

MGIT: Mycobacterium growth indicator tube; MTB: Mycobacterium tuberculosis; NALC-NaOH: N-acetyl-I-cysteine-sodium citrate- $\mathrm{NaOH}$; NTRL: National TB reference laboratory; RIF: Rifampicin

\section{Acknowledgements}

None.

\section{Authors' contributions}

SA conceived the study, supervised data collection, co-analysed the data and drafted of the manuscript; LK collected data, contributed to data analysis and assisted in drafting and submission of the manuscript. NMW, TK, JW, SJO, PO, MK, CA, and SM participated in data collection and review of the manuscript. LC contributed to data analysis, drafting and critical revision of the manuscript. All authors approved the final version of the manuscript.

\section{Funding}

None.

\section{Availability of data and materials}

All data contained within the article and its Additional file 1.

\section{Ethics approval and consent to participate}

The study was approved by the Kenyatta National Hospital institutional ethic review committee. This was retrospective study using remnant sputum samples and the consent of patients to participate was not required.

\section{Consent for publication}

Not applicable.

\section{Competing interests}

The authors declare that they have no competing interests.

\section{Author details}

${ }^{1}$ School of Physical and Biological Sciences, Maseno University, Kisumu, Kenya. ${ }^{2}$ National Public Health Laboratories, Nairobi, Kenya. ${ }^{3}$ Amref Health Africa, Nairobi, Kenya. ${ }^{4}$ Kenya Medical Research Institute, Nairobi, Kenya. ${ }^{5}$ Strathmore University, Nairobi, Kenya. ${ }^{6}$ National AIDS and STI Control Program, Nairobi, Kenya. ${ }^{7}$ Kisii teaching and Referral Hospital, Nairobi, Kenya.
Received: 8 May 2019 Accepted: 13 September 2019

Published online: 15 October 2019

\section{References}

1. World Health Organization. Global tuberculosis report 2016. 2016.

2. WHO. WHO Global Tuberculosis Report 2014. 2014. doi: WHO/HTM/TB/2014.08

3. Kwamanga D, Chakaya J, Sitienei J, Kalisvaart N, L'Herminez R, Van Der Werf MJ. Tuberculosis transmission in Kenya: results of the third National Tuberculin Survey. Int J Tuberc Lung Dis. 2010;14(6):695.

4. Enos M, Sitienei J, Ong'ang'o J, Mungai B, Kamene M, Wambugu J, et al. Kenya tuberculosis prevalence survey 2016: challenges and opportunities of ending TB in Kenya. PLoS One. 2018;13(12):e0209098. https://doi.org/10. 1371/journal.pone.0209098.

5. Helb D, Jones M, Story E, Boehme C, Wallace E, Ho K, et al. Rapid detection of Mycobacterium tuberculosis and rifampin resistance by use of ondemand, near-patient technology. J Clin Microbiol. 2010;48:229-37. https:// doi.org/10.1128/JCM.01463-09.

6. Wang $\mathrm{H}$, Chunyan Zhao FL. Rapid Identification of Mycobacterium tuberculosis Complex By a Novel Hybridization. Braz J Microbiol. 2011;42: 964-972. https://doi.org/10.1590/S1517-838220110003000016.

7. Centers for Disease Control and Prevention (CDC). CDC Grand Rounds: the TB/HIV syndemic. MMWR Morb Mortal Wkly Rep. 2012;61:484-9 doi: mm6126a3 [pii]

8. Woods GL. Mycobacterial susceptibility testing and reporting: when, how and what to test. Clin Microbiol Newsl. 2005;27(9):67. https://doi.org/10. 1016/S0196-4399(05)80022-4.

9. A.M. VT, P. B, J. A. C, A. C. T, J. D. J, D. M. B-M, et al. Optimizing prevention strategies and processes to reduce the impact of malaria on U.S. military forces. Am J Trop Med Hyg. 2012. p 9-30

10. WHO. Global tuberculosis report 2016. 2016. doi: ISBN 9789241565394.

11. Meaza A, Kebede A, Yaregal Z, Dagne Z, Moga S, Yenew B, et al. Evaluation of genotype MTBDRplus VER 2.0 line probe assay for the detection of MDRTB in smear positive and negative sputum samples. BMC Infect Dis. 2017;17: 280. https://doi.org/10.1186/s12879-017-2389-6.

12. Aljohani S, Alshomrani M. P285: comparison of two nucleic acid amplification assays, the probetec et assay and xpert mtb/rif assay, for detection of mycobacterium tuberculosis in respiratory specimens. Antimicrob Resist Infect Control. 2013;2:P285. https://doi.org/10.1186/20472994-2-s1-p285.

13. Raizada N, Sachdeva KS, Chauhan DS, Malhotra B, Reddy K, Dave PV, et al. A multi-site validation in India of the line probe assay for the rapid diagnosis of multi-drug resistant tuberculosis directly from sputum specimens. PLoS One. 2014;9:e88626. https://doi.org/10.1371/journal.pone.0088626.

14. Al-Darraji HAA, Razak HA, Ng KP, Altice FL, Kamarulzaman A. The diagnostic performance of a single GeneXpert MTB/RIF assay in an intensified tuberculosis case finding survey among HIV-infected prisoners in Malaysia. PLoS One. 2013;8(9):e73717. https://doi.org/10.1371/journal.pone.0073717.

15. Evans CA. Genexpert-a game-changer for tuberculosis control? PLoS Med. 2011;8:e1001064. https://doi.org/10.1371/journal.pmed.1001064.

16. Singh UB, Pandey P, Mehta G, Bhatnagar AK, Mohan A, Goyal V, et al. Genotypic, phenotypic and clinical validation of genexpert in extrapulmonary and pulmonary tuberculosis in India. PLoS One. 2016;11: e0149258. https://doi.org/10.1371/journal.pone.0149258.

17. Pandey P, Pant ND, Rijal KR, Shrestha B, Kattel S, Banjara MR, et al. Diagnostic accuracy of GeneXpert MTB/RIF assay in comparison to conventional drug susceptibility testing method for the diagnosis of multidrug-resistant tuberculosis. PLoS One. 2017;12:e0169798. https://doi. org/10.1371/journal.pone.0169798.

18. Pandey S, Congdon J, Mclnnes B, Pop A, Coulter C. Evaluation of the GeneXpert MTB/RIF assay on extrapulmonary and respiratory samples other 
than sputum: a low burden country experience. Pathology. 2017;49:70-4 https://doi.org/10.1016/j.pathol.2016.10.004.

19. Drobniewski F, Cooke M, Jordan J, Casali N, Mugwagwa T, Broda A, et al. Systematic review, meta-analysis and economic modelling of molecular diagnostic tests for antibiotic resistance in tuberculosis. Health Technol Assess (Rockv). 2015;19:1-188. https://doi.org/10.3310/hta19340.

20. Minion J, Pai M. Bacteriophage assays for rifampicin resistance detection in Mycobacterium tuberculosis: updated meta-analysis. Int J Tuberc Lung Dis. 2010;14:941-51.

21. Faburay AK, Mendy FS, Otu J, Faal-Jawara TI, Gehre F, Secka O. Performance comparison of lowenstein-jensen $(\mathrm{L})$ media supplemented with pyruvate, glycerol and a combination of both to assess the growth of Mycobacterium tuberculosis complex. Am J Respir Crit Care Med. 2017;195:A2080. https://doi. org/10.1164/ajrccm-conference.2017.A61.

22. Luetkemeyer AF, Kendall MA, Wu X, Lourenço MC, Jentsch U, Swindells S, et al. Evaluation of two line probe assays for rapid detection of mycobacterium tuberculosis, tuberculosis (TB) drug resistance, and non-TB mycobacteria in HIV-infected individuals with suspected TB. J Clin Microbiol. 2014;52:1052-9. https://doi.org/10.1128/JCM.02639-13.

23. Singh BK, Sharma SK, Sharma R, Sreenivas V, Myneedu VP, Kohli M, et al. Diagnostic utility of a line probe assay for multidrug resistant-TB in smearnegative pulmonary tuberculosis. PLoS One. 2017;12:e0182988. https://doi. org/10.1371/journal.pone.0182988.

24. Lin HC, Perng CL, Lai YW, Lin FG, Chiang CJ, Lin HA, et al. Molecular screening of multidrug-resistance tuberculosis by a designated public health laboratory in Taiwan. Eur J Clin Microbiol Infect Dis. 2017;36:2431-9. https://doi.org/10.1007/s10096-017-3082-9

25. Theron G, Peter J, Richardson M, Warren R, Dheda K, Steingart KR. GenoType MTBDRsl assay for resistance to second-line anti-tuberculosis drugs. Cochrane Database Syst Rev. 2016;2016. https://doi.org/10.1002/ 14651858.CD010705.pub3.

26. Pang $Y$, Dong $H$, Tan $Y$, Deng $Y$, Cai $X$, Jing $H$, et al. Rapid diagnosis of MDR and XDR tuberculosis with the MeltPro TB assay in China. Sci Rep. 2016;6: 25330. https://doi.org/10.1038/srep25330.

27. Malbruny B, Le Marrec G, Courageux K, Leclercq R, Cattoir V. Rapid and efficient detection of Mycobacterium tuberculosis in respiratory and nonrespiratory samples. Int J Tuberc Lung Dis. 2011;15:553-5. https://doi.org/10. 5588/ijtld.10.0497.

28. Ritu S, Jyoti A, Prabha L, Manpreet B, Myneeedu BP, Digamber B. Comparison of line probe assay with liquid culture for rapid detection of multi-drug resistance in Mycobacterium tuberculosis. Indian J Med Res. 2012;136:1044-7.

29. Tomasicchio M, Theron G, Pietersen E, Streicher E, Stanley-Josephs D, Van Helden P, et al. The diagnostic accuracy of the MTBDRplus and MTBDRsl assays for drug-resistant TB detection when performed on sputum and culture isolates. Sci Rep. 2016;6:17850. https://doi.org/10.1038/srep17850

30. Albert H, Bwanga F, Mukkada S, Nyesiga B, Ademun JP, Lukyamuzi G, et al. Rapid screening of MDR-TB using molecular line probe assay is feasible in Uganda. BMC Infect Dis. 2010;10:41. https://doi.org/10.1186/1471-2334-10-41.

31. Rufai SB, Kumar P, Singh A, Prajapati S, Balooni V, Singh S. Comparison of xpert MTB/RIF with line probe assay for detection of rifampin-monoresistant mycobacterium tuberculosis. J Clin Microbiol. 2014;52:1846-52. https://doi. org/10.1128/JCM.03005-13.

32. Yadav RN, Singh BK, Sharma SK, Sharma R, Soneja M, Sreenivas V, et al. Comparative evaluation of GenoType MTBDRplus line probe assay with solid culture method in early diagnosis of multidrug resistant tuberculosis (MDR-TB) at a tertiary Care Centre in India. PLoS One. 2013;8:e72036. https:// doi.org/10.1371/journal.pone.0072036.

33. Jain A, Singh PK, Chooramani G, Dixit P, Malhotra HS. Drug resistance and associated genetic mutations among patients with suspected MDR-TB in Uttar Pradesh, India. Int J Tuberc Lung Dis. 2016;20:870-5. https://doi.org/10. 5588/ijtld.15.0874

34. Liu Z, Pan A, Wu BB, Zhou L, He H, Meng Q, et al. Feasibility of a new model for early detection of patients with multidrug-resistant tuberculosis in a developed setting of eastern China. Tropical Med Int Health. 2017;22: 1328-33. https://doi.org/10.1111/tmi.12934.

35. Mironova S, Kontsevaya I, Pimkina E, Nikolayevskyy V, Skenders G, Kummik T, et al. Performance of the GenoType ${ }^{\circledast}$ MTBDRplus assay for detection of Mycobacterium tuberculosis drug resistance in routine settings: a multicentre study. Clin Microbiol Infect. 2011;17:S588.
36. Gersh J, Matemo D, Kinuthia J, Feldman Z, Dawson J, LaCourse S, et al. Evaluation of novel screens for pulmonary TB in people living with HIV in Kenya. Am J Respir Crit Care Med Conf Am Thorac Soc Int Conf ATS. 2018; 197:A5558.

37. Davis JL, Ho C, Cattamanchi A, Grinsdale J, Metcalfe JZ, Pandori M, et al. The clinical and public health impact of automated nucleic acid testing for TB evaluation in San Francisco. Am Thorac Soc. 2011:A5314. https://doi.org/10. 1164/ajrccm-conference.2011.183.1_meetingabstracts.a5314.

\section{Publisher's Note}

Springer Nature remains neutral with regard to jurisdictional claims in published maps and institutional affiliations.
Ready to submit your research? Choose BMC and benefit from:

- fast, convenient online submission

- thorough peer review by experienced researchers in your field

- rapid publication on acceptance

- support for research data, including large and complex data types

- gold Open Access which fosters wider collaboration and increased citations

- maximum visibility for your research: over $100 \mathrm{M}$ website views per year

At BMC, research is always in progress.

Learn more biomedcentral.com/submissions 